\title{
A rare case: small bowel intussusception in adult due to intraabdominal lipoma
}

\author{
Jansen Hatuaon Pasaribu*, Budi Laraswati \\ jr.jansenhp.gmail.com \\ Department of Radiology, Dr. Soetomo General Hospital, Surabaya, Indonesia \\ *Corresponding author
}

\begin{abstract}
Summary
Bowel obstruction caused by intussusception is a rare case. A non-typical sign makes the patient undergo several radiological examinations to establish diagnosis. From various intussusception causes, lipoma is a very rare cause. We reported a rare case of small bowel intussusception caused by lipoma who underwent ultrasonography (USG) and computed tomography (CT) scan examinations in Dr. Soetomo General Hospital, Surabaya, Indonesia.
\end{abstract}

Keywords: Intussusception, Lipoma, Ultrasonography, CT scan

\section{Introduction}

In the United States, mechanical bowel obstruction contributes $15 \%$ of emergency cases for abdominal pain complaint. The number is estimated to be more than 300,000 cases per year. It also contributes to approximately 30,000 deaths and high cost of medical care. Together, small and large bowel obstruction contribute to estimated $20 \%$ of acute abdominal surgical cases. (Gore et al., 2015). Out of all underlying causes, intussusception only accounts for $1-5 \%$ of bowel obstruction cases. (Marsicovetere et al., 2017)

In adults, intussusception is less common than in children and occurs more in small bowel compare to colon. The clinical symptoms and signs of adult intussusception could be non-typical, which present as bowel obstruction signs (abdominal pain, nausea, vomiting, change in bowel habits, etc). The non-typical nature of the clinical presentation requires several radiological examinations to establish diagnosis. Plain abdominal $\mathrm{x}$ ray (BOF) could be performed initially. Ultrasonography (USG) is widely accepted as a beneficial imaging tool. However, computed tomography (CT) is deemed the most important and sensitive diagnostic tool to establish intussusception diagnosis (Panzera et al., 2021; Lianos et al., 2013; Marsicovetere et al., 2017)

The most frequent underlying cause of adult intussusception is benign neoplasm (50-75\% of cases), which includes lipomas, hemangioma, lymphoid hyperplasia, leiomyomas, and polyps. (Marsicovetere et al., 2017) Histopathological examination could be performed to determine the exact cause.

\section{Case Report}

A 53 years old woman with a chief complaint of non-typical right abdominal pain since 4 hours before hospital admission. The patient also complained nausea and vomiting with whitish liquid vomit. No blood or dark liquid was found in the patient's vomit. The patient still managed to pass gas and defecate three times in a day with no blood or mucus.

Plain chest x-ray was performed with no presence of abnormality. BOF was also performed on the patient. 
Ground glass opacity (GGO) was observed from right hypochondria to right iliac region, which resulted in a shift of bowel gas shadow to the left side with minimal distribution at pelvic cavity (Figure 1).

Further examinations were performed, which were abdominal USG and CT scan with contrast. Abdominal USG revealed soft tissue mass with a size of $\pm 9 \times 3.6 \mathrm{~cm}$ at right lumbar region, which presented as pseudokidney sign on longitudinal view and as target sign on transversal view (Figure 2). These findings led to suspicion of intussusception. Abdominal CT scan supported ileocolic intussusception finding with a size of $\pm 9.2 \times 5.9 \times 10.5 \mathrm{~cm}$. Ileum, ileocaecum, caecum, and mesenteric pericaecum were the intussuseptum, and proximal ascending colon and subcentimeter lymph nodes at mesenteric pericaecal were the intussuscipien. No discrete mass was found in the abdominal cavity (Figure 3 ).

The patient underwent surgery and ileocolic intussusception was found with ileum, ileocaecum, and caecum as intussuseptum. A polyp mass was also found with a diameter of $\pm 4 \mathrm{~cm}$ at $10 \mathrm{~cm}$ from proximal ileocaecal junction (terminal ileum). Peritoneal fluid was clear. Terminal ileum and transversal colon resection and end-to-end anastomosis were performed. After surgery, histopathological examination was performed and revealed lipomas in colon.

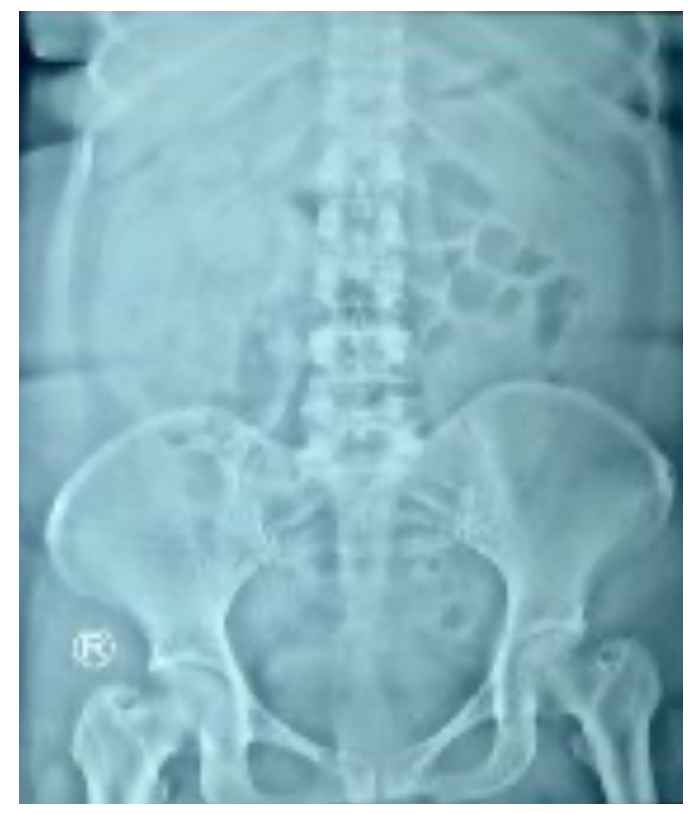

Figure 1. BOF of patient with GGO finding 


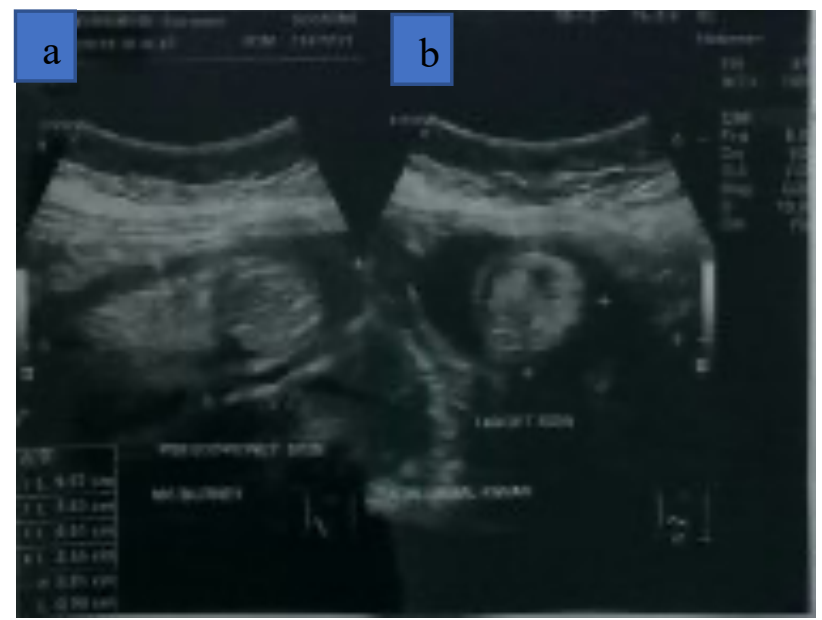

Figure 2. USG of patient. Pseudokidney sign (a) and target sign (b) led to suspicion of intussusception

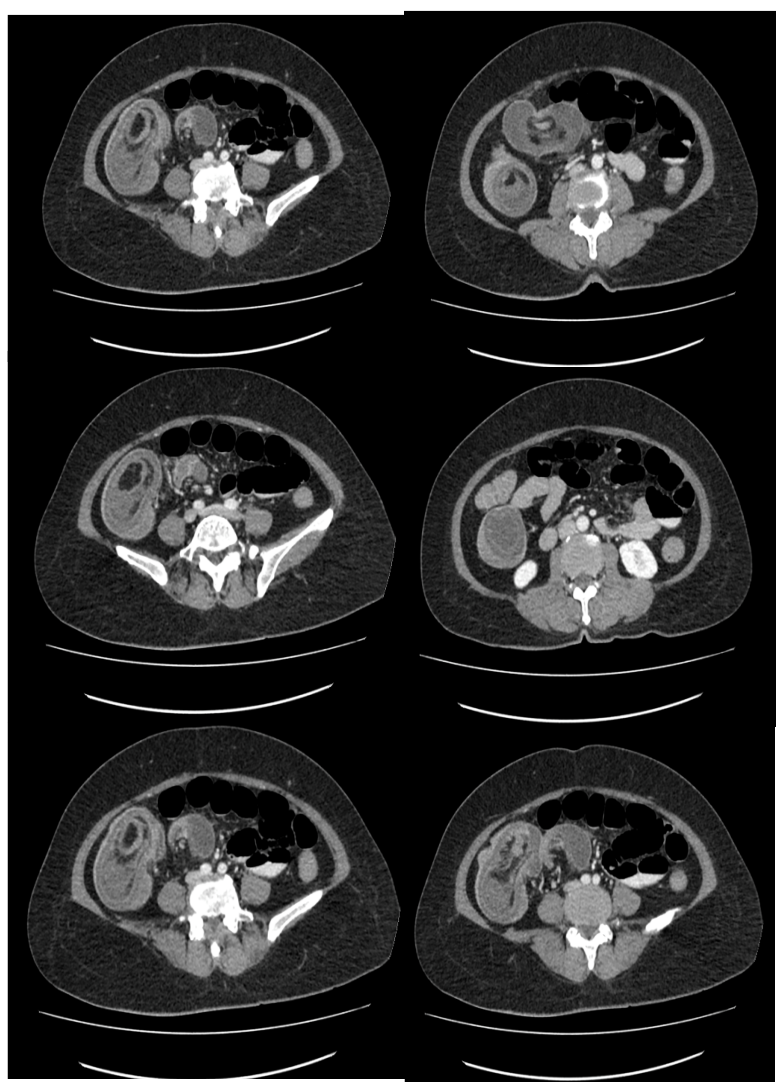

Figure 3. Abdominal CT scan supported intussusception finding 


\section{Discussion}

Cases of intussusception in adults are less frequent than children with an incidence of 1-5\%. The mean age of adult intussusception incidence is 50 years. Clinical symptoms of intussusception in adults are not typical with signs of bowel obstruction. Abdominal pain is the most common chief complaint and is accompanied with other associated complaint such as nausea, vomiting, obstipation, gastrointestinal bleeding, bowel habit changes, constipation, or bloating (Marsicovetere et al., 2017) In the current case, a 53 years old woman came to the hospital with a chief complaint of right abdominal pain. The patient also complained nausea and vomiting. The patient's complaints were non-specific, therefore additional examinations were required.

A gradual radiological examination is very important to help establish a diagnosis in cases of abdominal pain. BOF is the first diagnostic tool that could be used, especially if bowel obstruction signs dominate the clinical findings. BOF could reveal intestinal obstruction findings such as massive distention of bowel loops, colonic gas absence, and also might reveal the obstruction of location. However, this tool lacks specificity and sensitivity to establish intussusception diagnosis. (Panzera et al., 2021; Lianos et al., 2013; Marsicovetere et al., 2017). In the current case, GGO was observed from right hypochondria to right iliac region, which resulted in a shift of bowel gas shadow to the left side with minimal distribution at pelvic cavity. These findings led to additional examinations (USG and CT scan) in this case.

USG sensitivity and specificity for establishing intussusception diagnosis might reach $100 \%$ in experienced radiologists, especially in children. However, it is less accurate in adults but still could reveal classic intussusceptions features. The classic features include target sign on transverse view and pseudokidney sign on longitudinal view. (Lianos et al., 2013; Marsicovetere et al., 2017). In the current case, abdominal USG revealed soft tissue mass with a size of $\pm 9 \times 3.6 \mathrm{~cm}$ at right lumbar region, which presented as pseudokidney sign on longitudinal view and as target sign on transversal view. These findings were classic features of intussusception.

In adults, CT scan is deemed the most sensitive and important diagnostic tool for establishing intussusception diagnosis given its non-specific clinical findings. (Lianos et al., 2013) In the current case, abominal CT scan supported ileocolic intussusception finding with a size of $\pm 9.2 \times 5.9 \times 10.5 \mathrm{~cm}$. Ileum, ileocaecum, caecum, and mesenteric pericaecum were the intussuseptum, and proximal ascending colon and subcentimeter lymph nodes at mesenteric pericaecal were the intussuscipien.

Most adult intussusception occurs in small bowel and less common in colon, which accounts 20 to $25 \%$ of all intussusception cases. The most frequent underlying cause of adult intussusception is benign neoplasm (50-75\% of cases), which includes lipomas, hemangioma, lymphoid hyperplasia, leiomyomas, and polyps. Although lipoma is a benign neoplasm, it is one of the less common cause of adult intussusception (Lee and Chloe, 2017; Marsicovetere et al., 2017) In this case, a surgical intervention was performed and ileocolic intussusception was found with ileum, ileocaecum, and caecum as intussuseptum. A polyp mass was also found $10 \mathrm{~cm}$ from proximal ileocaecal junction (terminal ileum). After surgery, histopathological examination was performed and revealed lipomas in colon.

\section{Conclusion}

Intussusception in adults is a difficult case to detect because its clinical signs are not spesific. Appropriate radiological examinations are necessary to determine its diagnosis, while the exact cause of intussusception must be determined by histopathological examination performed after surgery. This means that interdisciplinary cooperation between radiologist, surgeons, and pathologists are important for patient management. 


\section{Acknowledgements}

The authors would like to thank Department of Radiology, General Surgery, and Anatomical Pathology of Dr. Soetomo General Hospital, Surabaya for providing data in this case report

\section{References}

Gore, R. M., Silvers, R. I., Thakrar, K. H., Wenzke, D. R., Mehta, U. K., Newmark, G. M., \& Berlin, J. W., 2015. Bowel obstruction. Radiologic Clinics, 53(6), 1225-1240.

Lee, D.E. and Choe, J.Y., 2017. Ileocolic intussusception caused by a lipoma in an adult. World Journal of Clinical Cases, 5(6), p.254.

Lianos, G., Xeropotamos, N., Bali, C., Baltogiannis, G., \& Ignatiadou, E., 2013. Adult bowel intussusception: presentation, location, etiology, diagnosis and treatment. Il Giornale di chirurgia, 34(9-10), 280.

Marsicovetere, P., Ivatury, S. J., White, B., \& Holubar, S. D., 2017. Intestinal intussusception: etiology, diagnosis, and treatment. Clinics in colon and rectal surgery, 30(01), 030-039.

Panzera, F., Di Venere, B., Rizzi, M., Biscaglia, A., Praticò, C.A., Nasti, G., Mardighian, A., Nunes, T.F. and Inchingolo, R., 2021. Bowel intussusception in adult: prevalence, diagnostic tools and therapy. World Journal of Methodology, 11(3), p.81. 